\title{
Anthropometric Characteristics and Mean Arterial Pressure in Preeclamptic and Normotensive Pregnant Women Visiting Antenatal Clinics: A Case Study in South Africa's Mthatha Area
}

\author{
Nokele, L.A. \\ Mammen, M. \\ Buga, G.A.B
}

Walter Sisulu University, P/Bag X1, UNITRA-5117, South Africa

Email:mmammen@wsu.ac.za

Doi:10.5901/mjss.2014.v5n20p2075

\section{Abstract}

This paper presents results from an analysis of patients' records of 711 pregnant women (14-40 year olds) between June 2002 and March 2012 and is a retrospective study. The study investigated anthropometric characteristics and mean arterial pressure in preeclamptic and normotensive pregnant women visiting antenatal clinics in South Africa's Mthatha area. BMI, WHR and MAP in Black African women in this area were carried out in order to fill a gap as data of association or correlation of such data on this population could not be located. The Research questions were: (a) Are MAP, BMI and WHR higher in preeclamptic women than in normotensive pregnant women in South Africa's Mthatha area? and, (b) Which one of the parameters (BMI, WHR and MAP) can be used as a strong predictor of occurrences of preeclampsia in this area? The data were statistically analysed. The main conclusions were that there were higher MAP, BMI and WHR in preeclamptic women than those who were not. Also, the study found a high correlation of these parameters in preeclamptic women. BMI correlated with DBP, MAP and WHR ( $r=0.43, r=0.38, r=0.44$, respectively), whereas WHR correlated with BMI and DBP only $(r=0.44,0.40$, respectively). Therefore, MAP, BMI and WHR may be used as predictors of preeclampsia. Between the three, BMI correlated more with both $D B P$ and MAP. Therefore, BMI may be a better predictor than WHR. Similar to other studies, we also found that age was a risk factor for preeclampsia and that waist circumference was higher in preeclamptic women.

Keywords: anthropometric characteristics, MAP, Preeclampsia

\section{Introduction}

The purpose of this research as part of a larger study was to investigate anthropometric characteristics and mean arterial pressure in preeclamptic and normotensive pregnant women visiting antenatal clinics in South Africa's Mthatha area. Researchers are aware that the cut-off values that denote medical risk in the Caucasian population cannot be extrapolated to other populations. Such observations are explicitly stated by researchers such as Kurpad, Tandon and Srinivasan (2003). No research data on anthropometric characteristics and mean arterial pressure in preeclamptic and normotensive pregnant women visiting antenatal clinics in Mthatha area in South Africa could be located. Measurements of Body Mass Index (BMI), Waist-to-Hip Ratio (WHR) and Mean Arterial Pressure (MAP) in Black African women in this area were carried out in order to fill the gap. The records of 711 pregnant women (14-40 year olds) who visited the Nelson Mandela Hospital and the surrounding Mthatha clinics over a 10 year period between June 2002 and March 2012 constituted the data. The research sought to find out (a) whether the MAP, BMI and WHR were higher in preeclamptic women than in normotensive pregnant women who visited Mthatha surrounding clinics and (b) which one of the parameters (BMI, WHR and MAP) can be used as a strong predictor of occurrences of preeclampsia in this region? The research objectives were (a) to compare the parameters such as MAP, BMI and WHR between preeclamptic women and normotensive pregnant women and (b) to find the association between MAP, BMI and WHR in the development of preeclampsia.

In order to maximise the availability of medical care to women generally and to rural women in particular, the South African health system has established numerous medical clinics in every district. These clinics are managed by nursing staff and are only peripherally supported by medical practitioners. This research report emanates from an analysis of patients' records for ten years and is a retrospective study through document analysis. In order to assist readers who are 
unfamiliar with some of the terms that are used in the study, the following terms are briefly explained below.

\subsection{Anthropometric characteristics}

Anthropometric characteristics refer to the measurement or description of the physical dimensions and properties of the body; typically, used on upper and lower limbs, neck, and trunk. In this study, only two anthropometric characteristics were investigated: the body mass index (BMI) and waist-to-hip ratio (WHR). The only other variable investigated was the mean arterial pressure (MAP).

\section{$1.2 B M I$}

$\mathrm{BMI}$ is a ratio between a person's weight and height and is calculated by dividing weight in $\mathrm{Kg}$ by height in meters squared. It is a measure of body fat based on height and weight of humans and provides a reliable indicator of body fatness for most people. Adult BMI calculator is available on the internet. However, the inferences drawn from the number are different for adults and amongst them different for men and women. Calculating BMI is an inexpensive and easy-toperform method of screening for weight categories that may lead to health problems.

According to William Reville Emeritus Professor of Biochemistry and Public Awareness of Science Officer at University College Cork, and the Science Today columnist for The Irish Times, BMI is not an infallible measure and states, advises people to calculate BMI but also check the ratio of waist-to-hip circumference and be ultra- conscious of abdominal fat (Reville, 2014). He sees BMI as a very crude index when applied to an individual as opposed to a population. One needs to become more discriminating in the use of $\mathrm{BMI}$ and more conscious of the health benefits of exercise. According to Reville (2014), BMI misses many people with excess body fat and warns that someone who had a normal BMI could still have too much internal fat and be prone to obesity-related illness.

\subsection{WHR}

WHR is considered as a more accurate way than BMI to assess individual fatness. There are two measurements needed and this can be done with a tape measure around the waist and around the hips. Divide the waist by the hip measurement. A ratio above 0.9 for men or 0.85 for women flags abdominal obesity and increased health risk (Reville, 2014).

\subsection{Preeclampsia}

Preeclampsia has been defined by many researchers (Brown et.al., 2001; ACOG Committee, 2002) to be characterized by blood pressure of $\geq 140 \mathrm{mmHg}$ systolic blood pressure (SBP) and $\geq 90 \mathrm{mmHg}$ diastolic blood pressure (DBP) and proteinuria of $\geq 1+$ dipstick in a previously normotensive women after the 20th week of gestation. This should be distinguished from severe preeclampsia. Preeclampsia affects $5-7 \%$ of all pregnancies and is the leading cause of maternal and fetal mortality and morbidity worldwide (Lana, 2004).

\subsection{Mean Arterial Pressure (MAP)}

The average MAP throughout the cardiac cycle is $95 \mathrm{mmHg}$ (Lowdermilk, 2004). The mean arterial pressure is determined by the stroke volume, with high stroke volume causing an increase in the mean arterial pressure and the decreased stroke volume also causing a decrease in the mean arterial pressure.

\subsection{Protenuria}

In normal pregnancy, proteinuria doesn't occur. Amino acids on the other hand may exceed their renal absorption capacity due to increased glomerular filtration rate and increased permeability of the glomerular basement membrane. This then leads to small amounts of protein of value less than $3 \mathrm{~g}$ in two readings taken at least 24 hours apart being lost in urine (Lowdermilk, 2004). 


\section{Research on Anthropometric Characteristics and their Association with other Factors}

Research has been ongoing on anthropometric characteristics and their association with other factors (see for example, Kurpad, Tandon and Srinivasan, 2003; Yalcin, Sahin and Yalcin, 2005; Tytmonas, 2006; Vazquez, Jacobs and Silventoinen (2007); Chen, Wang, Chang, Liu \& Chen, 2009; Gandhi, Koley and Sandhu, 2010; Lin, Lung, Liu, Jian, Ko, Huang, Ho, Chen, Chiang and Liaw (2013). Kurpad, Tandon and Srinivasan (2003) examined the correlation of waist circumference, hip circumference and waist-to-hip ratio with body mass Index. They carried out anthropometric measurements in a hospital-based, cross-sectional study in India on 285 subjects (207 men, mean age 37 years, range 18-76 years; and 78 women, mean age 31.5 years, range 18-69 years). The subjects comprised patients under psychiatric care as well as normal individuals. The prevalence of abdominal obesity using waist circumference was compared to the prevalence of abdominal obesity using waist-to-hip ratio using both the standard and the new recommended cut-offs for Asians. Their results were as follows: waist circumference correlated better with BMI than WHR (in men $r=0.821$ and 0.341 , and in women $r=0.729$ and $0.113 ; p<$ or $=0.01$ ). Forty-seven per cent of overweight men and $73 \%$ of overweight women had abdominal obesity as defined by the standard waist circumference cut-offs compared to none of the men and $47 \%$ of overweight women using the waist-to-hip ratio. The study concluded that waist circumference correlated better with BMI than WHR. The prevalence of abdominal obesity using waist circumference was found to be higher than that with waist-to-hip ratio. This was especially so in Asian Indians as the new recommendations of both body mass index and waist circumference cut-offs to detect subjects at medical risk were lower in them than in Caucasians. Yalcin, Sahin and Yalcin (2005) investigated anthropometric measurements which are most closely related to elevated blood pressure. According to them, "Epidemiological studies find a progressive increase in the prevalence of elevated blood pressure with increasing adipose tissue. But there is no common opinion about which effectiveness of the anthropometric measurement tools indicating general or android obesity are most important to follow up in patients with elevated blood pressures" (Yalcin, Sahin and Yalcin, 2005: 541). They chose a cross-sectional descriptive study of 1727 subjects of [894 men and 833 women, aged 18-65 years old in Edirne, Turkey. They measured each subject's weight, height, waist and hip circumference, triceps skin fold and blood pressures and calculated the waist to hip ratio and body mass index. The relations between blood pressure and different anthropometric variables in both genders were investigated in linear regression models. The reported results indicated that the mean systolic and diastolic blood pressures were $123.49+/-17.60$ and $78.79+/-10.37 \mathrm{mmHg}$. According to body mass index $23.7 \%$ of the subjects were obese $\left(.29 .9 \mathrm{~kg} / \mathrm{m}^{2}\right)$. When waist circumference cut-off points were compared with WHR, the android obesity ratio was doubled (32.3\% versus 16.6\%). 119 subjects (6.8\%) were not obese according to BMI but had waist circumference measurements above the cut-off points, suggesting a high cardiovascular risk. In the linear regression models, waist circumference was found to be an independent risk factor for blood pressure in men; however body mass was more important index and waist circumference somewhat less so for women. They concluded that in primary care, waist circumference should be a useful tool screening for and following android obesity in patients with elevated blood pressure.

Tytmonas (2006) researched the influence of increased BMI and abdominal obesity on the development of metabolic syndrome. The main respondents were patients of the Clinic of Family Medicine at Kaunas University of Medicine Hospital and the respondents were selected by stratified random sampling. A total of 2,774 patients (1,321 men and 1,453 women) were divided into groups by 10-year intervals from 20 to 70 and more years of age. There were 430 patients selected randomly in every age group. Abdominal obesity was estimated measuring waist circumference or after measuring the waist/hip ratio. BMI was calculated for all the patients. The survey results were used to estimate a correlation between increased BMI and waist circumference and detect the relation of BMI with the other factors. Amongst men, $75 \%$ with $\mathrm{BMI}>30$ had a waist circumference of $102 \mathrm{~cm}$ or greater and amongst women, $85 \%$ with BMI $>30$ had a waist circumference of $88 \mathrm{~cm}$ or greater. They found a statistically significant correlation between the waist circumference and WHR (in $70.2 \%$ of patients, with waist circumference of more than $102 \mathrm{~cm}$, the WHR was higher than 0.9 and in $82.2 \%$ of female patients with waist circumference of more than $88 \mathrm{~cm}$, the WHR was higher than 0.85 ). A positive family anamnesis of diabetes mellitus was documented in more than a half of the respondents who had the increased waist circumference (in $58.9 \%$ of men and $67.3 \%$ of women). The conclusion was that there was a statistically significant correlation between abdominal obesity, WHR, waist circumference and BMI and abdominal obesity was the mostly frequent indicator of metabolic syndrome.

\section{Research Methodology}

This was a retrospective study through document analysis. The study population was selected from records of pregnant 
women who visited the Nelson Mandela Hospital and the surrounding Mthatha clinics between June 2002 and March 2012. The age of the participants ranged from 14-40 years. Participants were requested to sign informed consent forms voluntarily and complete a questionnaire regarding their pregnancy and medical history. Informed consent forms were made available in both the local language isiXhosa and English. Each participant was dealt with during her visit during the period to which the data refer. In total, there were 711 pregnant women in this study. Participants were divided into 2 groups, namely; the normotensive pregnant women being the controls and preeclamptic women being the cases. The preeclamptic women were again divided into two groups, the first group was conveniently selected from the clinics (preeclamptic group 1) and the second group was taken straight from the ward where patients were identified as preeclamptics by specialist doctors (preeclamptic group 2). In preeclamptic group 1, participants were recruited before 20 weeks of pregnancy and they were followed up and those who turned into preeclamptic were selected. Women with systolic blood pressure $120 \pm 20 \mathrm{mmHg}$ and diastolic blood pressure of $80 \pm 10 \mathrm{mmHg}$ and no protein in urine were considered normotensive and those with blood pressure $\geq 140 / 90 \mathrm{mmHg}$ and $\geq 1+$ dipstick protein were considered preeclamptic. Information about the pregnancy outcomes was taken from the labor and delivery records taken by professional nurses. Those whose data were excluded from this study were women who were diabetic, those with chronic hypertension and other medical disorders, and preeclamptic women with a systolic blood pressure of $160 \mathrm{mmHg}$ and diastolic blood pressure of $110 \mathrm{mmHg}$ (severe preeclampsia).

Weight was measured in kilograms $(\mathrm{kg})$ using a digital scale. Participants were asked to take off all the heavy clothes, shoes and accessories before standing on the scale to measure weight. For measuring height, a stadiometer was used. Participants were asked to take off their shoes and stand straight in a lateral position against the vertical part of the stadiometer (ruler). A horizontal part of the stadiometer was then adjusted to rest on top of the participants' heads and readings were taken. The readings were recorded with height measured in meters $(\mathrm{m})$. BMI is a product of weight in kilograms divided by height in meters square and therefore it was calculated from the recorded measurements of weight and height.

For measuring the waist circumference, participants were asked to stand up still with their feet together, place their hands on the sides and to breathe gently. The inferior margin of the last rib and the crest of the llium were located to find the midpoint. The tape measure was then placed on the located midpoint with participants relaxing their stomachs without sucking in their stomachs though. It was made sure that the tape measure was parallel to the floor and that it does not compress the skin. A reading on the tape measure was taken and that was the waist circumference and the measurement was recorded. For measuring the hip circumference, participants were also asked to stand up still with their feet together. The tape measure was placed on the maximum part of their hip region/buttocks. Participants were asked to place their hands on the sides and to breathe gently. It was made sure that the tape measure was parallel to the floor. A reading from the measuring tape was taken and the hip circumference was then measured and the measurement was recorded. For checking for the presence of protein in urine, a dipstick was used and a measure of $\geq 1+$ in dipstick was considered as proteinuria.

Blood pressure was measured in $\mathrm{mmHg}$ by a sphygmomanometer. When measuring the blood pressure, participants were asked to sit down, relax and rest for about 10 minutes in an environment with no noise, no distractions and under room temperature before the measurements were made. After the rest and relaxation, an un-inflated cuff was placed around the arm above the elbow and at the height of the heart and a start button was pressed to inflate the cuff. The cuff was inflated and both the systolic blood pressure and diastolic blood pressure readings were displayed on the display of the sphygmomanometer and readings were recorded by the lead researcher.

\section{Data Analysis}

Data collected were analyzed statistically using STATISTICA version 5 for Microsoft Windows. The parameters of the 3 groups were compared using ANOVA. Pearsons coefficient ( $r$ ) was used for correlating the parameters collected. P value $<0.05$ was considered significant.

\section{Results}

There were a total of 711 participants (663 from the clinics and 48 recruited from the wards). Out of the 663 from the clinics, 601 remained normal pregnant women and 62 became preeclamptic before delivery and the latter group constitued preeclamptic group 1. About $10 \%$ of the followed up participants became preeclamptics. The 48 preeclamptics who were recruited straight from the ward after being identified by specialist doctors as preeclamptics constituted preeclamptic group 2. The results and discussion refer to the preeclamptic groups as group 1 and group 2. 
Ages of the participants ranged from 14-49 years. Figure 1 shows the mean ages of the groups with their standard deviations. The mean ages across the studied groups were significant to one another $(P<0.05)$, with preeclamptic women showing to be older than the normal pregnant women. Normal pregnant women group was significantly different in age from the preeclamptic group from 1 with $P=0.01$, normal pregnant women were significantly different in age from the preeclamptic group 2 women with $\mathrm{P}=0.00$ and preeclamptic group 1 were significantly different preeclamptic group 2 with $\mathrm{P}=0.00$.

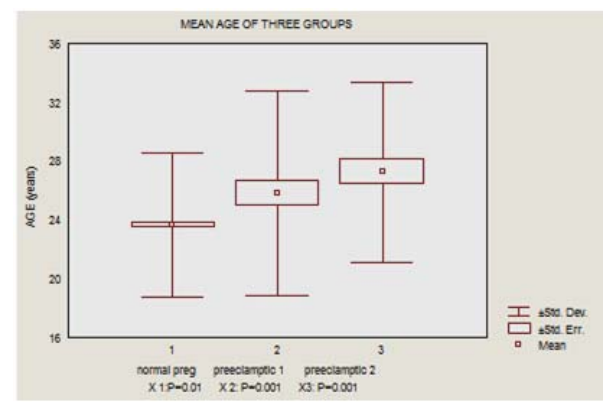

Figure 1: Ages of participants presented according to their groups as mean \pm standard deviation. $\times_{1}$ : Comparison between normal pregnant and preeclamptic group $1 ; \times_{2}$ : Comparison between preeclamptic group 1 and preeclamptic group 2; $\mathrm{x}_{3}$ : Comparison between normal pregnant and preeclamptic group 2.

\subsection{Data Collected At Recruitment}

Note: In all the Tables below, the following abbreviations apply: WHR: waist-hip ratio, BMl: body mass index, SBP: systolic blood pressure, DBP: diastolic blood pressure, MAP: mean arterial pressure, wks: weeks, kg: kilograms, m: meter, $\mathrm{kg} / \mathrm{m}^{2}$ : kilograms per square meter, $\mathrm{cm}$ : centimetres)

Table 1: Anthropometric Measurements Compared Between Normal Pregnant Women And Preeclamptic Group 1

\begin{tabular}{lccc}
\hline Variables & Normal pregnant N=601 & Preeclamptic 1 N=62 & P-value \\
\hline Waist circumference $(\mathrm{cm})$ & $88.91 \pm 11.32$ & $99.13 \pm 11.10$ & $<0.05$ \\
Hip circumference $(\mathrm{cm})$ & $102.05 \pm 11.35$ & $107.50 \pm 9.67$ & $>0.05$ \\
WHR & $0.87 \pm 0.97$ & $0.92 \pm 0.89$ & $<0.05$ \\
Weight $(\mathrm{kg})$ & $67.30 \pm 13.03$ & $73.46 \pm 13.93$ & $<0.05$ \\
Height $(\mathrm{m})$ & $1.57 \pm 0.70$ & $1.58 \pm 0.67$ & $>0.05$ \\
BMl $\left(\mathrm{kg} / \mathrm{m}^{2}\right)$ & $27.18 \pm 5.14$ & $29.07 \pm 4.56$ & $<0.05$ \\
Protein at recruitment & $0.10 \pm 0.44$ & $0.58 \pm 0.92$ & $<0.05$ \\
Gestation at recruitment (wks) & $18.17 \pm 3.83$ & $21.82 \pm 5.70$ & $<0.05$ \\
SBP at recruitment $(\mathrm{mmHg})$ & $113.11 \pm 12.15$ & $147.50 \pm 17.32$ & $<0.05$ \\
DBP at recruitment $(\mathrm{mmHg})$ & $70.37 \pm 9.14$ & $99.26 \pm 8.71$ & $<0.05$ \\
MAP $(\mathrm{mmHg})$ & $84.62 \pm 8.90$ & $104.61 \pm 15.87$ & $<0.05$ \\
\hline
\end{tabular}

(Values are mean \pm standard deviation; $<0.05$ : statistically significant; $>0.05$ : statistically non-significant)

Table 1: Waist circumference, WHR, weight, BMI, protein in urine, gestation age, SBP, DBP, MAP, last SBP and last DBP at recruitment were all significantly different across the normal pregnant group and preeclamptic group 1, with the preeclamptic group 1 having higher levels of each one of these variables than the normal pregnant women. Hip circumference and height were not significantly different across these groups. 
Table 2: Anthropometric Measurements Between Normal Pregnant And Preeclamptic Group 2

\begin{tabular}{lccc}
\hline Variables & Normal pregnant N=601 & Preeclamptic 2 N=48 & P-value \\
\hline Waist circumference $(\mathrm{cm})$ & $88.91 \pm 11.32$ & $99.33 \pm 11.65$ & $<0.05$ \\
Hip circumference $(\mathrm{cm})$ & $102.05 \pm 11.35$ & $100.16 \pm 13.48$ & $<0.05$ \\
WHR & $0.87 \pm 0.97$ & $1.00 \pm 0.13$ & $<0.05$ \\
Weight $(\mathrm{kg})$ & $67.30 \pm 13.03$ & $76.14 \pm 15.94$ & $<0.05$ \\
Height $(\mathrm{m})$ & $1.57 \pm 0.70$ & $1.55 \pm 0.12$ & $<0.05$ \\
BMl $\left(\mathrm{kg} / \mathrm{m}^{2}\right)$ & $27.18 \pm 5.14$ & $32.44 \pm 11.68$ & $<0.05$ \\
Protein at recruitment & $0.10 \pm 0.44$ & $0.92 \pm 1.01$ & $<0.05$ \\
Gestation at recruitment $(\mathrm{wks})$ & $18.17 \pm 3.83$ & $22.32 \pm 5.86$ & $<0.05$ \\
SBP at recruitment $(\mathrm{mmHg})$ & $113.11 \pm 12.15$ & $153.94 \pm 20.21$ & $<0.05$ \\
DBP at recruitment $(\mathrm{mmHg})$ & $70.37 \pm 9.14$ & $101.83 \pm 12.80$ & $<0.05$ \\
MAP $(\mathrm{mmHg})$ & $84.62 \pm 8.90$ & $109.17 \pm 20.12$ & $<0.05$ \\
\hline
\end{tabular}

(Values are mean \pm standard deviation; $<0.05$ : statistically significant; $>0.05$ : statistically non-significant)

Table 2: Waist circumference, hip circumference, WHR, weight, height, BMI, protein in urine, gestation age, SBP, DBP, MAP, last SBP and last DBP at recruitment were all significantly different across the groups, with the preeclamptic group 2 having higher levels of each one of these variables than the normal pregnant women except hip circumference and height of preeclamptic group 2 which were lower than that of the normal pregnant women.

Table 3: Anthropometric Measurements Between Preeclamptic Group 1 And Preeclamptic Group 2

\begin{tabular}{lccc}
\hline Variables & Preeclamptic 1 N=62 & Preeclamptic 2 N=48 & P-value \\
\hline Waist circumference $(\mathrm{cm})$ & $99.13 \pm 11.10$ & $99.33 \pm 11.65$ & $>0.05$ \\
Hip circumference $(\mathrm{cm})$ & $107.50 \pm 9.67$ & $100.16 \pm 13.48$ & $<0.05$ \\
WHR & $0.92 \pm 0.89$ & $1.00 \pm 0.13$ & $<0.05$ \\
Weight $(\mathrm{kg})$ & $73.46 \pm 13.93$ & $76.14 \pm 15.94$ & $>0.05$ \\
Height $(\mathrm{m})$ & $1.58 \pm 0.67$ & $1.55 \pm 0.12$ & $>0.05$ \\
BMl $\left(\mathrm{kg} / \mathrm{m}^{2}\right)$ & $29.07 \pm 4.56$ & $32.44 \pm 11.68$ & $>0.05$ \\
Protein at recruitment & $0.58 \pm 0.92$ & $0.92 \pm 1.01$ & $>0.05$ \\
Gestation at recruitment $(\mathrm{wks})$ & $21.82 \pm 5.70$ & $22.32 \pm 5.86$ & $>0.05$ \\
SBP at recruitment $(\mathrm{mmHg})$ & $147.50 \pm 17.32$ & $153.94 \pm 20.21$ & $>0.05$ \\
DBP at recruitment $(\mathrm{mmHg})$ & $99.26 \pm 8.71$ & $101.83 \pm 12.80$ & $>0.05$ \\
MAP $(\mathrm{mmHg})$ & $104.61 \pm 15.87$ & $109.17 \pm 20.12$ & $>0.05$ \\
\hline
\end{tabular}

(Values are mean \pm standard deviation; $<0.05$ : statistically significant; $>0.05$ : statistically non-significant)

Table 3: Only hip circumference and WHR were significantly different between preeclamptics group 1 and 2. Waist circumference, weight, BMI, protein in urine, gestation age, SBP, DBP, MAP, last SBP and last DBP at recruitment were all non-significant across these groups.

\subsubsection{Correlation Of Parameters In Normal Pregnant Women}

When we looked at the association of some parameters in normal pregnant women, we found a significant correlation between parity and weight ( $r=0.14)$. Age correlated with weight and BMI $(r=0.36 ; r=0.31$, respectively). As expected, BMI correlated with weight $(r=0.87)$. BMI also correlated with SBP, DBP, MAP $(r=0.13 ; r=0.13 ; r=0.15$, respectively) and had a negative correlation with height $(r=-0.23)$. There was a negative correlation between gestation age at recruitment and height $(r=0.19)$. MAP correlated with SBP and DBP $(r=0.81 ; r=0.92$, respectively). MAP also correlated with weight and BMI ( $r=0.16 ; r=0.15$, respectively).

\subsubsection{Correlation Of Parameters In Preeclamptic Group I}

The results of this study showed that, when the age increases, the WHR also increases ( $r=0.46)$. WHR also correlated with parity $(r=0.70)$. BMI only significantly correlated with weight $(r=0.89)$ in this group of participants. Just like in the 
normal pregnant group, an increase in MAP was associated with an increase in SBP and DBP $(r=0.89 ; r=0.95$, respectively).

At 20 weeks of gestation the preeclamptic group 1 had no significant correlation between the parameters as the normotensive pregnant women group had except the correlation between age and WHR $(0=0.46)$ as well as between parity and WHR ( $r=0.70)$.

\subsubsection{Correlation Of Parameters Of Preeclamptic Group 2}

In this population group, the age significantly correlated with weight $(r=0.50)$ but not with BMI. There was a correlation between parity and weight ( $r=0.64)$. We found that WHR correlated with the weight, BMI and DBP $(r=0.46 ; r=0.44 ; r=0.40$, respectively). BMI correlated with weight, as expected ( $r=0.93)$. BMI also correlated with MAP and DBP $(r=0.38 ; r=0.43$, respectively). The results showed correlation between gestation age at recruitment and protein record at recruitment $(r=0.53)$. The MAP showed positive correlation with weight $(r=0.42)$.

\section{Discussion}

The study included 711 participants, 601 of whom had normal pregnancies, 62 in preeclamptic group 1 and 48 in preeclamptic group 2. The mean maternal age for all 3 groups differed significantly with an increased maternal age in preeclamptic group 1 and group $2(\mathrm{P}=0.00)$. These results showed that with increasing age, the risk of preeclampsia increased. Ventura et.al (2001), Luealon et.al (2010) and Lamminppa et.al (2012) also found increased maternal age as a risk factor for developing preeclampsia. Lamminppa et.al (2012) reported that this can be a result that advanced maternal age (AMA) women were more often obese than the ones younger than them and as we have stated before, many researchers found obesity as a risk factor for preeclampsia. Lamminppa et.al (2012) assumed that this can also be related to the aging of the uterine blood vessels. Luealon (2010) also put similar explanations as Lamminppa et.al (2012). Lueanol and Phupong (2010) also explained that it may be related to the damage of the vascular endothelium occurring with maternal aging and also related to atherosis which causes obstruction of the lumen of maternal spiral arteries.

Out of 601 normal pregnant women, $62 \%$ were primipara and 38\% were multipara. Out of 62 preeclamptic group 1 women, 36\% were primipara and 64\% were multipara. Out of 48 preeclamptic group 2 women, $27 \%$ were primipara and $73 \%$ were multipara. Therefore, in this study preeclamptic women were mostly multiparous. However, findings in studies by Hernandez-Diaz, et.al (2009), El-Moselhy et.al (2011) and Tebeu et.al (2011) showed primigravida and nulliparity as a risk of preeclampsia.

In this study we found MAP to be significantly higher in preeclamptic women than the normal pregnant women and Emilija and Vladimir's (2011a; 2011b) also had the same findings. However, unlike Emilija and Vladimir (2011a; 2011b) who found only MAP as a good predictor of preeclampsia, we found both SBP, DBP and MAP to be good predictors for preeclampsia. The explanation to increased SBP, DBP and in turn MAP, according to Shah (2005) may be due to the increased sensitivity of preeclamptic women to angiotensin II (ANG II) as a result of change in conformation of the angiotensin II type-1 (AT1) receptors. The increased sensitivity to ANG II may then result to increased aldosterone release, increased smooth muscle vasoconstriction, increased $\mathrm{Na}$ reabsorption and water retention and therefore increased blood pressure and blood volume leading to high BMI. ANG II increases NADPH oxidase, superoxide production and NO scavenging, this way decreasing NO and in turn decreasing the vasodilation effects of NO (Schalkwijik \& Stehouwer, 2005). This may therefore lead to increased peripheral resistance and in turn increased blood pressure, be it SBP, DBP or MAP. In addition to decreased vasodilator effects of NO, increased blood pressure can also slightly be caused by the decrease in angiotensin-(1-7), which is a vasodilator that is said to be decreased in preeclamptic women (Kobori et.al, 2007; Irani \& Xia, 2011).

In normal pregnant women there was a weak positive correlation ( $r=0.15)$ between MAP and BMI. However, there was no correlation between MAP and WHR.

It was observed that in preeclamptic women, WHR increased with increasing maternal age and parity. The findings showed that preeclamptic women have higher BMI and WHR when compared with normal pregnant women. These findings state that, as BMI and WHR increase, women stand high risks of developing preeclampsia. The findings about BMI support those of other researcher's findings, namely, Sattars et.al (2001), Baeten et.al (2001) Ramsay et.al (2003) and Bodnar et.al (2005). Therefore, from this study also BMI and WHR may be used as predictors for preeclampsia because these parameters in preeclamptic group 2 both correlated with DBP and BMI also correlated with MAP. As mentioned earlier, these preeclamptic group 2 women were confirmed as preeclamptics by specialist doctors. However, Cnossen et.al (2007) in his study found BMI as a weak predictor for preeclampsia while Shamsi et.al (2010) found no 
association between BMI and risks of preeclampsia.

In this study, preeclamptic women showed relatively high waist circumference when they were compared to the normal pregnant women. According to Sattar et.al (2001), the waist circumference can be used as a predictor only before the $16^{\text {th }}$ week of gestation. However, we found a higher waist circumference after the $16^{\text {th }}$ week of gestation. The significant difference of waist circumference between preeclamptic group 1 and 2 may be due to the differences in their gestation age. The increase in the hip and waist circumferences in accordance to the increase in gestational age may be due to the increased fat deposits and water retention in preeclamptic women more than normal pregnant women which leads to an increase in WHR. Yamamoto et.al (2001), also found a higher WHR in preeclamptic women than in normal pregnant women.

\section{Conclusions}

This study found a higher MAP, BMI and WHR in preeclamptic women. We also found a high correlation of these parameters in preeclamptic women. BMI correlated with DBP, MAP and WHR ( $r=0.43, r=0.38, r=0.44$, respectively), whereas WHR correlated with BMI and DBP only $(r=0.44,0.40$, respectively). Therefore, MAP, BMI and WHR may be used as predictors of preeclampsia. Between the three, BMI correlated more with both DBP and MAP. Therefore, BMI may be a better predictor than WHR. As in other studies, we also found that age was a risk factor for preeclampsia and that waist circumference was higher in preeclamptic women.

\section{Recommendations}

Overweight, obese and women with high waist circumference need to be advised to lose weight when they are planning to conceive. Women also need to be advised to be cautious about the negative effects and risks involved when having children at much older ages.

\section{References}

ACOG Committee on practice Bulletins-Obstetrics. 2002. Diagnosis and management of preeclampsia and eclampsia. Obstet Gynecol., 99(1): 159-167.

Baeten, J.M., Bukusi, E.A. \& Lambe, M. 2001. Pregnancy complications and outcomes among overweight and obese nulliparous women. Am J Public Health, 91: 436-40.

Bodnar, L.M., Ness, R.B., Markovic, N. \& Roberts, J.M. 2005. The risk of preeclampsia with increasing pre-pregnancy body mass index. Ann Epidemiol, 15(7): 475-82.

Brown, M.A, Lindheimer, M.D., de Swiet, M., van Assche, A. \& Moutquin, J-M. 2001. The classification and diagnosis of the hypertensive disorders of pregnancy: statement from the International Society for the Study of Hypertension in Pregnancy (ISSHP). Hypertension in Pregnancy; 20 (1): IX-XIV.

Chen, C.C., Wang, W.S., Chang, H.Y., Liu, J.S. \& Chen, Y.J. 2009. Heterogeneity of body mass index, waist circumference, and waistto-hip ratio in predicting obesity-related metabolic disorders for Taiwanese aged 35-64 years. Clin Nutr., 28(5):543-548.

Cnossen, J.S., Leeflang, M.M., de Haan, E.E., Mol, B.W., van der Post, J.A., Khan, K.S., \& ter Reit, G. 2007. Accuracy of body mass index in predicting preeclampsia: bivariate meta-analysis. BJOG. An International Journal of Obstetrics and Gynaecology, 114 (12): 1477-1485.

El-Moselhy, E.A., Khalifa, H.O., Amer, S.M., Mohammad, K.I. \& Abd El-Aal, H.M. 2011. Risk factors and impact of preeclampsia: An epidemiological study among pregnant mothers in Cairo, Egypt. Journal of American Science, 7 (5): 311-323. http://www.americanscience.org Retrieved April 22, 2014.

Emilija, J. \& Vladimir, J. 2011a. Prediction of mild and severe preeclampsia with blood pressure measurements in first and second trimester of pregnancy. PZU Medica, 82: 845-850.

Emilija, J. \& Vladimir, J. 2011b. Demographic characteristics in preeclamptic women in Mecedonia. Rev Med Chile, 139: 748-754. http://www.scielo.cl/pdf/rmc/v139n6/art08.pdf Retrieved Jan 12, 2014.

Gandhi, M., Koley, S., \& Sandhu, J.S. 2010. Association between anthropometric characteristics and physical strength in school going children of Amritsar. The Anthropologist,12 (1): 35-39.

Hernandez-Diaz, S., Toh, S. \& Cnattingius, S. 2009. Risk of preeclampsia in first and subsequent pregnancies: Prospective cohort study. BMJ, 338: b2255. http://www.bmj.com/content/338/bmj.b2255 Retrieved Jan 30, 2013.

Irani, R.A. \& Xia, Y. 2011. Renin angiotensin signaling in normal pregnancy and preeclampsia. NIH public access, 31(1): 47-58

Kobori, H., Nangaku, M., Navar, L.G. \& Nishiyama, A. 2007. The intrarenal renin-angiotensin system: From physiology to the pathobiology of hypertension and kidney disease. Pharmacological reviews, 59 (3): 251-287.

Kurpad, S.S., Tandon, H. \& Srinivasan, K. 2003. Waist circumference correlates better with body mass index than waist-to-hip ratio in Asian Indians. Natl Med J India, 16(4):189-92. 
Lana, KW. (2004). Diagnosis and management of preeclampsia. Am Fam Physician, 70(20): 2317-24.

Lamminpaa, R., Venvilainen-Julkunen, K., Gissle,r M. \& Heinonen, S. 2012. Preeclampsia complicated by advanced maternal age: A registry based study on primiparous women in Finland 1997-2008. BMC Pregnancy and child birth, 12: 47.

Lin, W.Y., Lung, C.C., Liu, T.S., Jian, Z.H., Ko. P.C., Huang, J.Y., Ho, C.C., Chen, S.C., Chiang, Y.C. \& Liaw, Y.P. 2013. The association of anthropometry indices with gout in Taiwanese men. BMC Endocr Disord,13(1):30.

Lowdermilk, D.L. \& Perry, S.E. 2004. Maternity \& Women's Health Care. $8^{\text {th }}$ edition. St. Louis:bMosby, Inc.

Luealon, P. \& Phupong, V. 2010. Risk factors of preeclampsia in Thai women. J Med Assoc Thai, 93(6): 661-666.

Ramsay, J.E., Jamieson, N., Greer, I.A. \& Sattar, N. 2003. Paradoxical elevation in Adiponectin concentrations in women with preeclampsia. Journal of the American Heart Association, 42: 891-894. https://hyper.ahajournals.org/content/42/5/891.abstract Retrieved August 11, 2009.

Reville, W. 2014. Body Mass Index is not an infallible measure. The Irish Times, May 22, 2014. http://www.irishtimes.com/news/science/ body-mass-index-is-not-an-infallible-measure-1.1804196. Retrieved May 25, 2014.

Sattar, N., Clark, P., Holmes, A., Lean, M., Walker, I. \& Greer, I. 2001. Antenatal waist circumference and hypertension risk. Obstet Gynecol, 97 (2): 268-271.

Schalkwijik, C.G. \& Stehouwer, C.D.A. 2005. Vascular complications in diabetes mellitus: the role of endothelial dysfunction. Clin Sci (Lond). 109 (2): 143-159.

Shah, D.M. 2005. Role of the renin-angiotensin system in the pathogenesis of preeclampsia. Am J Physiol Renal Physiol, 288: F614F625.

Shamsi, U., Hatcher, J., Shams, A., Zuberi, N., Qadri, Z. \& Saleem, S. 2010. A multicentre matched case control study of risk factors for preeclampsia in healthy women in Pakistan. BMC Women's Health, 10:14

Tebeu, P.M., Foumane, P., Mbu, R., Fosso, G., Biyaga, P.T. \& Fomulu, J.N. 2011. Risk factors for hypertensive disorders in pregnancy: A report from the Maroua Regional Hospital, Cameroon. J Repro Infertil, 12(3): 227-234

Tytmonas, G. 2006. The influence of increased body mass index and abdominal obesity on the development of metabolic syndrome. Medicina, 42(2):123-129.

Vazquez, G., Duval, S., Jacobs, D.R. \& Silventoinen, K. 2007. Comparison of body mass index, waist circumference, and waist/hip ratio in predicting incident diabetes: a meta-analysis. Epidemiol Rev., 29:115-128. http://www.ncbi.nim.nih.gov/pubmed/17494056 , 2013.

Ventura, S.J., Martin, J.A., Menacker, F. \& Hamilton, B.E. 2001. Births: Final data for 1999. National Vital Statistics Reports, 49 (1). http://www.cdc.gov/nchs/data/nvsr/nvsr49/nvsr49_01.pdf Retrieved June 14, 2009.

Yalcin, B.M., Sahin, E.M. \& Yalcin, E. 2005. Which anthropometric measurements is most closely related to elevated blood pressure? Fam Pract., 22(5):541-547.

Yamamoto, S., Douchi, T., Yoshimitsu, N., Nakae, M. \& Nagata, Y. 2001. Waist to hip circumference ratio as a significant predictor of preeclampsia, irrespective of overall adiposity. J Obstet Gynecol Res, 27(1); 27-31. 